\title{
Diet and Cancer Prevention Research: From Mechanism to Implementation
}

\author{
Johanna W. Lampe \\ Public Health Sciences Division, Fred Hutchinson Cancer Research Center, Seattle, WA, USA
}

\begin{abstract}
Between $30 \%$ to $50 \%$ of cancer cases are estimated to be preventable through reduced exposure to tobacco, occupational carcinogens, and infectious agents, and adoption of lifelong healthy eating and a physically active lifestyle. In the past, diet and cancer prevention research has aimed to understand the effects of specific foods and nutrients on cancer-related mechanisms. More recently, there has been a shift in emphasis toward a more holistic focus on patterns of diet, reflecting the goal to understand the impact of adhering to broader public health recommendations. It is increasingly apparent from observational studies that different patterns of diet and physical activity are manifest in a metabolic state that is more, or less, conducive to the acquisition of genetic and epigenetic alterations leading to carcinogenesis. Experimental studies in cell systems, animals and humans have expanded our understanding of the many mechanisms by which specific dietary constituents may modulate inflammation and immune function, carcinogen metabolism, hormone and growth-factor regulation, DNA repair capacity, cell-cycle control, and proliferation and apoptosis. However, few mechanistic studies in animal models have evaluated diets containing the complex mixtures that make up human diets. Overall, more studies are needed across the continuum of prevention research, from basic mechanistic research on the effects of diet patterns on fundamental biologic processes to studies testing the efficacy of implementing lifestyle-directed cancer prevention strategies.
\end{abstract}

Key Words Cancer, Prevention, Diet, Implementation science, Intervention study

\section{INTRODUCTION}

The global burden of cancer is high and is increasing. Cancer contributes to one in eight deaths worldwide and has overtaken cardiovascular disease as the leading cause of death in many parts of the world [1]. Given the costs and challenges of treatment, identifying and adopting strategies for cancer prevention are becoming a necessity. Between 30\% to 50\% of all cancer cases are estimated to be preventable through reduced exposure to tobacco, occupational carcinogens, and infectious agents, and adoption of lifelong healthy eating and a physically active lifestyle [2]. In 2018, the World Cancer Research Fund (WCRF) and American Institute for Cancer Research (AICR) published the Third Expert Report, Diet, Nutrition, Physical Activity and Cancer: A Global Perspective [3]. An important aspect of this latest report is a shift in emphasis from effects of specific foods and nutrients to a more holistic focus on patterns of diet and physical activity. Although public health recommendations typically include these broader messages, research is often lacking in direct support of this complexity.

Diet is a complex exposure composed of myriad potentially beneficial and harmful compounds that are rarely consumed in isolation, but rather as part of a matrix or pattern. It is becoming increasingly apparent that different patterns of diet and physical activity manifest in a metabolic and hormonal state that is more, or less, conducive to the acquisition of genetic and epigenetic alterations. These alterations can lead to cellular structural and functional changes described in part by the hallmarks of cancer [4].

Cancer prevention research relies on a continuum of research methods to address the effects of diet and constituents of diet (Fig. 1). Population-based, longitudinal cohort studies are an important approach to identify risk factors for cancer. A group (i.e., cohort) of unaffected individuals are well-characterized at baseline with regard to diet, physical activity, occupational and lifestyle factors and are followed prospectively, typically for decades, for cancer outcomes. The 


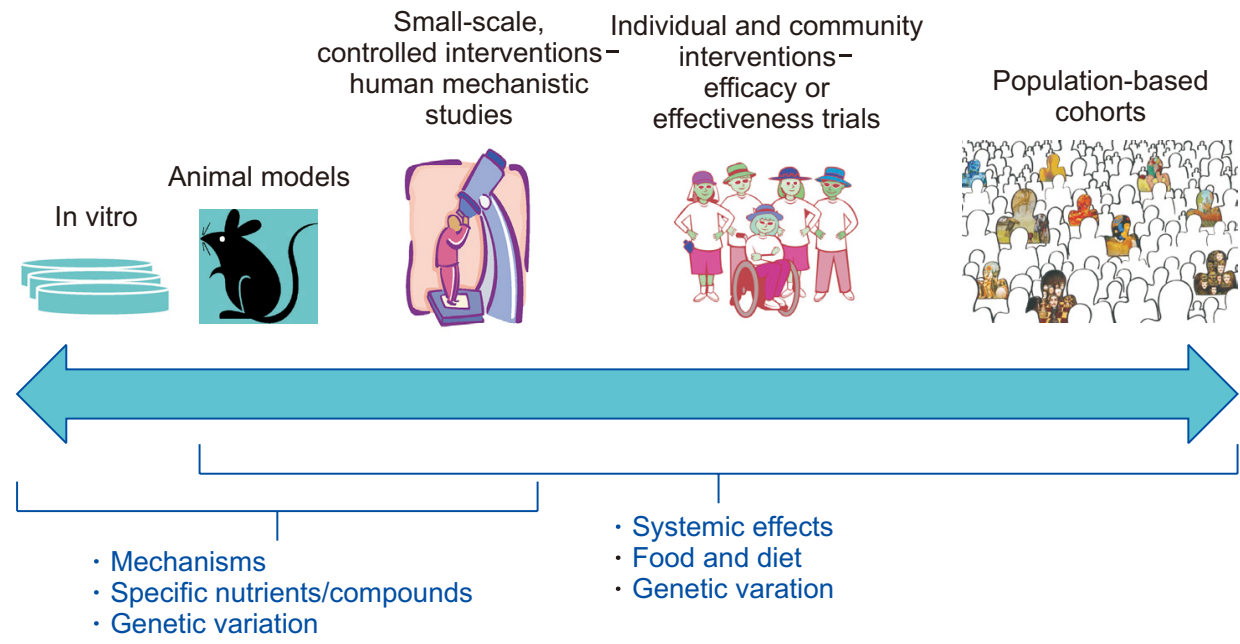

Small-scale, Individual and community ontrolled interventions- interventionsstudies effectiveness trials
Figure 1. A continuum of research approaches is used for studying diet and cancer prevention. Populationbased cohorts followed longitudinally identify risk factors for cancer in humans. Diets, foods or dietary constituents associated with cancer risk in cohort studies can be tested experimentally in animals to evaluate their effect on tumorigenesis. Mechanisms identified in cell systems and animals can be followed up in human interventions and cohort studies using biomarkers of cancer-risk pathways. The impact of diet-gene interactions can be tested, both experimentally and observationally, across the research continuum. findings from such observational studies are critical drivers of the experimental cancer prevention studies in animal models and humans. Foods or dietary constituents associated with cancer risk in cohort studies can be studied experimentally in animals to evaluate their effect on tumorigenesis. Similarly, mechanistic findings in cell systems and animals generate hypotheses tested in human interventions and cohort studies. For example, genetic variants in cells and rodent models may alter response to a dietary compound; such gene-by-diet interactions can be tested in humans, both experimentally and observationally $[5,6]$.

\section{MECHANISTIC STUDIES OF DIET AND CANCER PREVENTION}

To date, experimental studies in cell systems, animals and humans have expanded our understanding of the many mechanisms by which dietary constituents may increase or decrease cancer risk. These include, but are not limited to, modulation of inflammation and immune function, carcinogen metabolism, hormone and growth-factor regulation, DNA repair capacity, cell-cycle control, and proliferation and apoptosis [7]. However, few mechanistic studies in animal models have evaluated diets containing the complex mixtures of compounds that make up human diets.

Most studies of diet and tumorigenesis in animal models use purified and semi-purified diets and modify amounts of one, or a few, specific nutrients or bioactives of interest at a time. Some studies have focused on combinatorial effects of nutrients on tumorigenesis, including testing factorially the interactions of several compounds [8]. In contrast, only a few studies of diet and cancer have fed human diets or diets with the equivalent level of complexity, to rodents [911]. Identifying approaches to conducting interventions in animals that more closely align with the complexity of dietary patterns in humans is important for moving forward the field [12]. More parallel, co-clinical studies in animals and humans that address the effects of dietary patterns on cancer risk are needed to characterize the biological mechanisms by which diet, nutrition, adiposity, and physical activity affect cancer processes [13].

Dietary interventions in humans can be divided into two major categories: (1) basic experimental studies with humans or "human mechanistic studies," and (2) individual and community-based intervention trials. The United States National Institutes of Health $(\mathrm{NIH})$ distinguishes between these different categories of studies and application of them. Human mechanistic studies are defined as "designed to understand a biological or behavioral process, the pathophysiology of a disease, or the mechanism of action of an intervention" [14]. These include dietary interventions to answer basic science questions about normal function or characterize mechanisms of response to an intervention-how an intervention works. In contrast, intervention trials evaluate the effects of an intervention on health-related biomedical or behavioral outcomes, with the objective of determining the clinical safety, tolerability, feasibility, efficacy and/or effectiveness of an intervention.

Human mechanistic studies of dietary patterns, often conducted as controlled feeding studies, are typically short-term interventions and rely on intermediate biomarkers (Fig. 2). We have applied these types of studies to understand the impact of several types of dietary patterns on metabolic and inflammation pathways important to cancer risk. With the goal to understand the physiologic effect of dietary acculturation, the process by which immigrants adopt the host-country diet and lifestyle, we tested whether a traditional Mexican diet, compared with a commonly consumed United States diet, would improve insulin sensitivity and lower biomarkers of low-grade chronic inflammation. In a randomized crossover feeding trial in first- and second-generation healthy women of Mexican descent, the Mexican diet improved glycemic control, while having no impact on biomarkers of inflammation [15]. Using a similar design, we have also tested the effects of feeding controlled low- and high-glycemic load diet pat- 


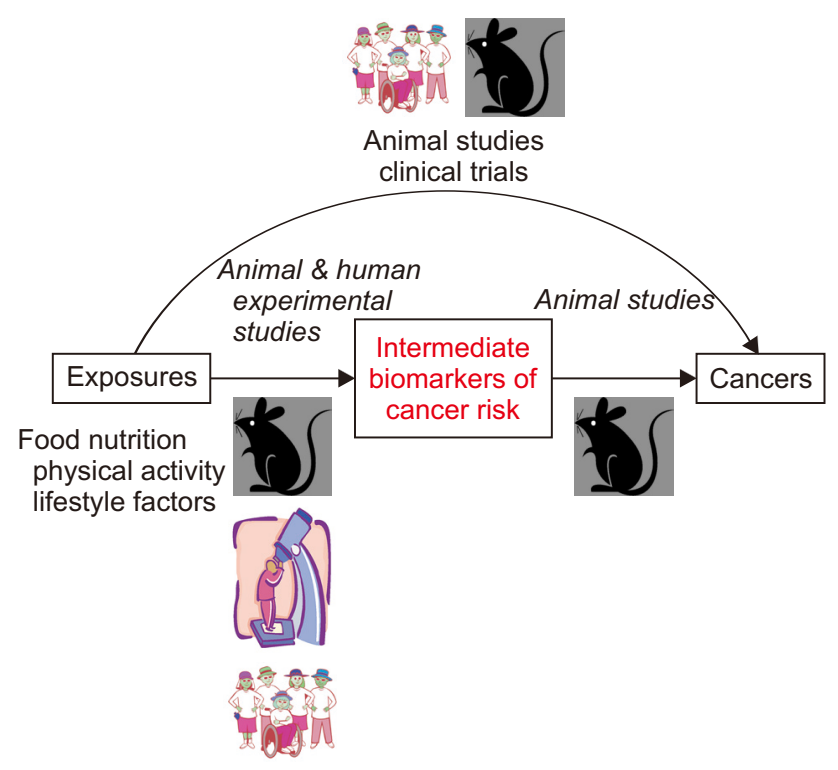

Figure 2. Animal and human studies contribute to bridging the knowledge gaps in experimental diet and cancer prevention research. Leveraging the use of intermediate biomarkers in animal studies with cancer endpoints can help to inform approaches in shortterm human interventions with intermediate measures and provide mechanistic support for diet-cancer relationships. Adapted from Lampe and Hursting, 2015 [13].

terns on cancer-risk biomarkers [16]. The low-glycemic load diet reduced serum C-reactive protein concentrations in participants with high fat mass and favorably altered tryptophan metabolism, as measured by metabolomics [17]. Tryptophan is a precursor for many biologically important metabolites involved in neurotransmission, redox reactions, and inflammation and immune responses. The low-glycemic load diet pattern, compared to the high-glycemic load pattern, reduced conversion of tryptophan to the proinflammatory branch of the kynurenine pathway [17].

\section{CLINICAL TRIALS OF DIET AND CANCER PREVENTION}

As defined by the Unites States $\mathrm{NIH}$, clinical trials are human research studies in which participants are prospectively assigned to one or more interventions (which may include a placebo or other control) to evaluate the effects of those interventions on health-related biomedical or behavioral outcomes. In relation to studies of cancer prevention, the outcomes could be a particular cancer (e.g., colon cancer), a precursor lesion (e.g., adenomatous polyp), or intermediate biomarkers of cancer risk (e.g., blood or stool-based biomarkers) (Fig. 2).

Experimental human studies of dietary patterns with cancer as the primary outcome are scarce. These types of studies need to be conducted over extended periods of time and therefore require behavioral interventions to change participants' diets. For example, the Women's Health Initiative Dietary Modification Trial, a controlled clinical trial, tested the hypothesis that a low-fat dietary pattern compared to a usual dietary pattern would reduce the risk of breast and colorectal cancers and coronary heart disease in postmenopausal women. Post-menopausal women ( $n=48,836$ ) were randomized to intervention or control and followed for an average of 8.1 years. A low-fat dietary pattern intervention did not result in a statistically significant reduction in the risk of invasive breast or colorectal cancer, although there were trends toward greater reduction in breast cancer $[18,19]$. Similarly, the PREDIMED (Prevención con Dieta Mediterránea) trial was designed to assess the intervention effects of two Mediterranean diets compared to a low-fat control diet on cardiovascular death, myocardial infarction and stroke (composite primary endpoint). Several secondary endpoints, including major cancers (colorectal, breast, lung, stomach, and prostate), were also evaluated. This study randomized 7,447 participants who were followed for a median of 4.8 years [20,21], but the study was stopped early, limiting study of the cancer outcomes. Nested studies using intermediate biomarkers of risk within trials such as PREDIMED have also provided a wealth of information on the effects of these diet patterns on inflammation and metabolism biomarkers (Fig. 2) [22].

Some cancers advance from detectable precursor lesions, such that reducing the number of precursor lesions can reduce cancer risk. For example, adenomatous polyps in the colon and rectum are a risk factor for colorectal cancer; removing the polyps lowers colorectal cancer risk [23]. By extension reducing development of polyps through lifestyle changes may lower cancer risk. To date, several counseling-based dietary interventions have attempted to reduce risk of colorectal adenoma recurrence, but intervention effects were not statistically significant [24-26].

The success of testing long-term intervention trials of cancer prevention requires participants to make diet and other lifestyle changes and adhere to them for years. Consequently, in the face of a sufficiently powered study of adequate duration, it is often unclear whether the null effects of a dietary intervention were due to a biological lack of effect or issues of participant adherence to the intervention. Several prospective cohort studies have shown that greater adherence to WCRF/ AICR Recommendations is associated with greater reduction in risk of certain cancers, cancer in general, and cancer mortality $[27,28]$, supporting strong biological underpinnings of the recommendations. Thus, in order to conduct clinical trials requiring lifestyle changes, a better understanding of how and why successful behavior change occurs is critical to develop effective and efficient behavior interventions to reduce cancer risk. 


\section{IMPLEMENTATION RESEARCH TO TEST PROGRAIIS AND POLICIES}

Substantial gaps also remain in cancer prevention implementation research. It has become evident that educating people about lifestyle factors that cause, or protect against, cancer and just making health recommendations are by themselves insufficient to bring about substantial, sustained changes in behavior. Implementing cancer prevention strategies requires programs and public health policies that encourage lifelong healthy eating and a physically active lifestyle. Thus, longterm, it is important to develop a framework for evaluating programs and policies to assess their impact and effectiveness $[29,30]$.

\section{SUMMARY}

We have made substantial progress in identifying the risks and benefits of components of diet in relation to certain cancers; however, gaps remain in diet and cancer prevention research. More studies are needed across the continuum of cancer prevention research, from basic mechanistic research on the effects of diet patterns on fundamental biologic processes to studies testing the efficacy of implementing lifestyle-directed cancer prevention strategies. We need to make use of parallel, co-clinical studies in animals and humans to further mechanistic understanding of effects of diet patterns on cancer-related pathways. We need to develop and test new behavior-change approaches to identify culturally relevant, effective ways to help individuals and communities adopt cancer-preventive lifestyles. Finally, we need to continue to implement research to test national and international programs and policies.

\section{ACKNOWLEDGMENTS}

This work was supported by United States NIH R01 CA192222 and P30 CA015704.

\section{CONFLICTS OF INTEREST}

No potential conflicts of interest were disclosed.

\section{REFERENCES}

1. Bray F, Ferlay J, Soerjomataram I, Siegel RL, Torre LA, Jemal A. Global cancer statistics 2018: GLOBOCAN estimates of incidence and mortality worldwide for 36 cancers in 185 countries. CA Cancer J Clin 2018;68:394-424.

2. World Health Organization (WHO). Cancer prevention. http:// www.who.int/cancer/prevention/en/. Accessed October 30, 2019.

3. World Cancer Research Fund, American Institute for Cancer Research. Continuous update project. Diet, nutrition, physical activity and cancer: a global perspective. A summary of the third expert report. London, World Cancer Research Fund International, 2018.

4. Hanahan D, Weinberg RA. Hallmarks of cancer: the next generation. Cell 2011;144:646-74.

5. Navarro SL, Chang JL, Peterson S, Chen C, King IB, Schwarz Y, et al. Modulation of human serum glutathione S-transferase A1/2 concentration by cruciferous vegetables in a controlled feeding study is influenced by GSTM1 and GSTT1 genotypes. Cancer Epidemiol Biomarkers Prev 2009;18:2974-8.

6. Wang LI, Giovannucci EL, Hunter D, Neuberg D, Su L, Christiani DC. Dietary intake of cruciferous vegetables, glutathione S-transferase (GST) polymorphisms and lung cancer risk in a Caucasian population. Cancer Causes Control 2004;15:977-85.

7. Chen KL, Jung P, Kulkoyluoglu-Cotul E, Liguori C, Lumibao J, Mazewski C, et al. Impact of diet and nutrition on cancer hallmarks. J Cancer Prev Curr Res 2017;7:240.

8. Newmark HL, Yang K, Kurihara N, Fan K, Augenlicht LH, Lipkin M. Western-style diet-induced colonic tumors and their modulation by calcium and vitamin D in C57BI/6 mice: a preclinical model for human sporadic colon cancer. Carcinogenesis 2009;30:88-92.

9. Thompson HJ, Neuhouser ML, Lampe JW, McGinley JN, Neil ES, Schwartz Y, et al. Effect of low or high glycemic load diets on experimentally induced mammary carcinogenesis in rats. Mol Nutr Food Res 2016;60:1416-26.

10. McDaniel SM, O'Neill C, Metz RP, Tarbutton E, StacewiczSapuntzakis M, Heimendinger J, et al. Whole-food sources of vitamin A more effectively inhibit female rat sexual maturation, mammary gland development, and mammary carcinogenesis than retinyl palmitate. J Nutr 2007;137:1415-22.

11. Rozen P, Liberman V, Lubin F, Angel S, Owen R, Trostler N, et al. A new dietary model to study colorectal carcinogenesis: experimental design, food preparation, and experimental findings. Nutr Cancer 1996;25:79-100.

12. Warden $\mathrm{CH}$, Fisler JS. Comparisons of diets used in animal models of high-fat feeding. Cell Metab 2008;7:277.

13. Lampe JW, Hursting SD. Integrating the mechanistic literature: toward co-clinical approaches in nutritional research. The Digest 2015;50:1-5.

14. National Institutes of Health (NIH). NIH plans for Clinical Trial Specific Parent R01 and Parent R21 Funding Opportunity Announcements. https://grants.nih.gov/grants/guide/notice-files/ NOT-OD-18-010.html. Accessed March 13, 2020.

15. Santiago-Torres M, Kratz M, Lampe JW, Tapsoba Jde D, Breymeyer KL, Levy L, et al. Metabolic responses to a traditional Mexican diet compared with a commonly consumed US diet in women of Mexican descent: a randomized crossover feeding trial. Am J Clin Nutr 2016;103:366-74.

16. Neuhouser ML, Schwarz Y, Wang C, Breymeyer K, Coronado $\mathrm{G}$, Wang $\mathrm{CY}$, et al. A low-glycemic load diet reduces serum C-reactive protein and modestly increases adiponectin in overweight and obese adults. J Nutr 2012;142:369-74.

17. Navarro SL, Tarkhan A, Shojaie A, Randolph TW, Gu H, Djukovic $D$, et al. Plasma metabolomics profiles suggest beneficial effects 
of a low-glycemic load dietary pattern on inflammation and energy metabolism. Am J Clin Nutr 2019;110:984-92.

18. Beresford SA, Johnson KC, Ritenbaugh C, Lasser NL, Snetselaar LG, Black HR, et al. Low-fat dietary pattern and risk of colorectal cancer: the Women's Health Initiative Randomized Controlled Dietary Modification Trial. JAMA 2006;295:643-54.

19. Prentice RL, Caan B, Chlebowski RT, Patterson R, Kuller LH, Ockene JK, et al. Low-fat dietary pattern and risk of invasive breast cancer: the Women's Health Initiative Randomized Controlled Dietary Modification Trial. JAMA 2006;295:629-42.

20. Toledo E, Salas-Salvadó J, Donat-Vargas C, Buil-Cosiales P, Estruch R, Ros E, et al. Mediterranean diet and invasive breast cancer risk among women at high cardiovascular risk in the PREDIMED trial: a randomized clinical trial. JAMA Intern Med 2015;175:1752-60.

21. Estruch R, Ros E, Salas-Salvadó J, Covas MI, Corella D, Arós $F$, et al. Primary prevention of cardiovascular disease with a Mediterranean diet supplemented with extra-virgin olive oil or nuts. N Engl J Med 2018;378:e34.

22. Casas R, Sacanella E, Urpí-Sardà M, Corella D, Castañer O, Lamuela-Raventos RM, et al. Long-term immunomodulatory effects of a Mediterranean diet in adults at high risk of cardiovascular disease in the PREvención con Dleta MEDiterránea (PREDIMED) randomized controlled trial. J Nutr 2016;146:1684-93.

23. Brenner H, Stock C, Hoffmeister M. Effect of screening sigmoidoscopy and screening colonoscopy on colorectal cancer incidence and mortality: systematic review and meta-analysis of randomised controlled trials and observational studies. BMJ 2014;348:g2467.

24. Schatzkin A, Lanza E, Corle D, Lance P, Iber F, Caan B, et al.
Lack of effect of a low-fat, high-fiber diet on the recurrence of colorectal adenomas. Polyp Prevention Trial Study Group. N Engl J Med 2000;342:1149-55.

25. MacLennan R, Macrae F, Bain C, Battistutta D, Chapuis $P$, Gratten $\mathrm{H}$, et al. Randomized trial of intake of fat, fiber, and beta carotene to prevent colorectal adenomas. J Natl Cancer Inst 1995;87:1760-6.

26. McKeown-Eyssen GE, Bright-See E, Bruce WR, Jazmaji V, Cohen LB, Pappas SC, et al. A randomized trial of a low fat high fibre diet in the recurrence of colorectal polyps. Toronto Polyp Prevention Group. J Clin Epidemiol 1994;47:525-36.

27. Vergnaud AC, Romaguera D, Peeters PH, van Gils $\mathrm{CH}$, Chan DS, Romieu I, et al. Adherence to the World Cancer Research Fund/American Institute for Cancer Research guidelines and risk of death in Europe: results from the European Prospective Investigation into Nutrition and Cancer cohort study. Am J Clin Nutr 2013;97:1107-20.

28. Romaguera D, Vergnaud AC, Peeters $\mathrm{PH}$, van Gils $\mathrm{CH}$, Chan DS, Ferrari P, et al. Is concordance with World Cancer Research Fund/American Institute for Cancer Research guidelines for cancer prevention related to subsequent risk of cancer? Results from the EPIC study. Am J Clin Nutr 2012;96:150-63.

29. World Health Organization (WHO). Cancer control: knowledge into action: WHO guide for effective programmes; module 2. https://www.who.int/cancer/publications/cancer_control_ prevention/en/. Accessed October 30, 2019.

30. Clinton SK, Giovannucci EL, Hursting SD. The World Cancer Research Fund/American Institute for Cancer Research Third Expert Report on Diet, Nutrition, Physical Activity, and Cancer: impact and future directions. J Nutr 2020;150:663-71. 